\section{Analgesia postoperatoria para craneotomías en pediatría: experiencia en un Hospital de Tercer Nivel}

\author{
Postoperative analgesia for craniotomies in pediatrics: \\ experience in a Third Level Hospital
}

Dra. Erika León-Álvarez, ${ }^{*}$ Dra. Janet Gloria Ortiz-Bautista, ${ }^{\ddagger}$

Dra. Columba Ortega-Munguía, ${ }^{\S}$ Dra. Chiharu Murataף

Citar como: León-Álvarez E, Ortiz-Bautista JG, Ortega-Munguía C, Murata C. Analgesia postoperatoria para craneotomías en pediatría: experiencia en un Hospital de Tercer Nivel. Rev Mex Anestesiol. 2021; 44 (2): 84-90. https://dx.doi.org/10.35366/99010

\begin{abstract}
RESUMEN. Introducción: Existe la creencia de que los pacientes no experimentan dolor intenso después de cirugía intracraneal. La estimulación simpática secundaria a dolor puede ocasionar hipertensión intracraneal y sangrado postoperatorio. Es controvertido el uso de opioides para analgesia postcraneotomías por temor a sus efectos colaterales que pueden enmascarar signos de alteración neurológica. En pediatría hay estudios limitados. Objetivo: Describir el nivel de control del dolor postcraneotomías al usar buprenorfina ketorolaco y ondansetrón en pacientes pediátricos. Métodos: Estudio de cohorte descriptivo. Incluimos niños de 0-17 años programados para cirugía intracraneal electiva. Para el control del dolor se administró buprenorfina, ketorolaco y ondansetrón en infusión por 30 horas. Se investigó dolor al iniciar la infusión a las cuatro, ocho, 12, 24 y 30 horas; variables hemodinámicas y grado de sedación. Resultados: 109 pacientes fueron incluidos. Se observó adecuado control del dolor en $71.56 \%, 28.4 \%$ tuvo control insuficiente con una diferencia estadísticamente significativa $(p<0.001)$. Hubo sedación moderada en $5.6 \%$ iniciando la infusión y a las 24 horas $(4.5 \%$ ) sin repercusión hemodinámica. Se detectó náusea en $8.2 \%$ y vómito en $6.64 \%$; no se presentó sedación profunda, ni depresión respiratoria. Conclusiones: Estos hallazgos sugieren que es una opción efectiva para tratar el dolor postcraneotomías en pediatría.
\end{abstract}

ABSTRACT. Introduction: There is still a belief that patients do not experience intense pain after intracranial surgery. Sympathetic stimulation associated with pain can lead to elevated intracranial pressure and postoperative haemorrhage. There is controversy about the use of opioids for postoperative analgesia in craniotomies, owing to fear of its side effects, which can mask signs of neurological alteration. There are limited studies in the pediatric patient for post-craniotomy analgesia. Objective: To describe the postcraneotomies pain control level, using buprenorphine in partnership with ketorolac and ondansetron in pediatric patients. Methods: Descriptive cohort study. For postoperative pain control, patients were given continuous infusion buprenorphine, ketorolac and ondansetron for 30 hours. The main variables to investigate were pain at beginning of infusion, at four, eight, 12, 24 and 30 hours, hemodynamic variables and depth of sedation. Results: 109 patients were included. Adequate control of pain was observed in $71.56 \%$ of patients, whereas in $28.4 \%$ insufficient control was found, with a statistically significant difference $(p<0.001)$. There was moderate sedation in $5.6 \%$ of the patients at the start of infusion and at 24 hours (4.5\%), without significant impact on hemodynamic variables. Nausea was found in $8.2 \%$ and vomiting in $6.64 \%$. No deep sedation, or respiratory depression was presented. Conclusions: These findings suggest that is an effective option to treat postcraneotomy pain in pediatric patients.

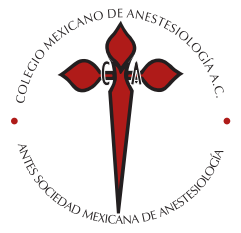

Palabras clave:

Buprenorfina, analgesia, ketorolaco, pediátricos, cirugía intracraneal, dolor.

Keywords:

Buprenorphine, analgesia, ketorolac, pediatric, intracranial surgery, pain.

* Neuroanestesióloga. Departamento de Anestesiología, Instituto Nacional de Pediatría, Cuidad de México.

${ }^{\ddagger}$ Anestesióloga Pediatra. Departamento de Anestesiología, Instituto Nacional de Pediatría, Cuidad de México.

$\S$ Anestesióloga Pediatra.

Departamento de Anestesiología, Hospital de Especialidades Pediátrico de León, Guanajuato, México. " Maestro en Rehabilitación Neurológica. Departamento de Metodología de la Investigación, Instituto Nacional de Pediatría, Ciudad de México.

Correspondencia: Erika León-Álvarez Uxmal Núm. 37-602, Col. Narvarte Poniente, 03020, Alcaldía Benito Juárez, Ciudad de México, Tel: 55 8557-6146

Recibido: 21-05-2020 Aceptado:02-02-2021

\section{INTRODUCCIÓN}

E l dolor es un síndrome complejo que causa angustia emocional y física, lo cual produce un impacto fisiológico adverso en varios órganos y sistemas, afectando la recuperación de los pacientes $^{(1)}$.

Aunque la manipulación del parénquima cerebral per se no es dolorosa, las bases anatómicas y fisiológicas de- muestran que el dolor postquirúrgico experimentado por los pacientes sometidos a craneotomías es de origen somático debido a la manipulación del escalpe (inervación de ramas originadas en el plexo cervical y el nervio trigémino), músculos pericraneales, tejido blando y duramadre (inervada por los nervios que acompañan a las arterias meníngeas) $(1,2)$. Sin embargo, el dolor en pacientes pediátricos después de craneotomías aún es subestimado, a causa de la creencia 
de que los pacientes no experimentan dolor intenso después de este tipo de cirugías.

La Clasificación Internacional de Trastornos de Cefalea publicado por la International Headache Society establece criterios diagnósticos para diferentes cefaleas, ha clasificado el dolor de cabeza postcraneotomía y lo ha subdividido en las variedades agudo y persistente ${ }^{(2)}$. Actualmente la evidencia muestra que alrededor de $60 \%$ de los pacientes postcraneotomías, experimentan dolor moderado a severo en el postoperatorio inmediato o presentan dolor persistente con elementos neuropáticos varios meses después (dolor crónico) ${ }^{(3)}$. En su estudio Bronco y colaboradores encontraron que, a pesar de la aplicación de analgesia multimodal, $16 \%$ de los pacientes neuroquirúrgicos pediátricos sufrieron dolor moderado a severo en la sala de recuperación y 6\% presentó dolor moderado a severo en el primer y segundo día postquirúrgico ${ }^{(4)}$.

La estimulación simpática asociada a dolor puede conducir a hipertensión arterial, agitación, temblores, vómito e incremento de la presión intracraneal, posibles eventos nocivos, principalmente por el riesgo de hemorragia intracraneal postoperatoria $^{(5,6)}$, reflejándose en una estancia hospitalaria prolongada que a su vez aumenta los gastos hospitalarios ${ }^{(7)}$.

Los opioides parenterales constituyen la piedra angular para el control del dolor moderado a severo, especialmente después de cirugía mayor. No obstante, existe controversia en su uso para analgesia postoperatoria en craneotomías debido al temor de sus efectos colaterales tales como sedación, depresión respiratoria, miosis y náusea, que pueden enmascarar signos de deterioro neurológico y alterar la valoración neurológica temprana, por lo que se requiere precaución en el manejo del dolor postoperatorio, a fin de no interferir en dicha valoración.

En pediatría el manejo del dolor es complejo, dadas las barreras que limitan la adecuada evaluación del dolor postoperatorio, como pueden ser la dificultad para reconocer, valorar y cuantificar la intensidad del dolor, debido a que los niños menores de cuatro años y aquéllos con ciertas afecciones neurológicas y/o intelectuales no son capaces de verbalizar o describir exactamente la magnitud de su $\operatorname{dolor}^{(8)}$.
En la literatura médica de la especialidad no encontramos ninguna guía de práctica clínica para analgesia postoperatoria en neurocirugía pediátrica. La mayoría de los estudios se han realizado en población adulta.

El objetivo de nuestro estudio es describir la calidad analgésica de buprenorfina en asociación con ketorolaco y ondansetrón, administrado en infusión continua intravenosa en niños sometidos a procedimientos neuroquirúrgicos intracraneales así como describir parámetros hemodinámicos, posible presencia de sedación profunda y eventos adversos.

\section{MÉTODOS}

Previa aprobación del Comité de Ética e Investigación del Instituto Nacional de Pediatría y obtención del consentimiento informado de los padres así como asentimiento informado en

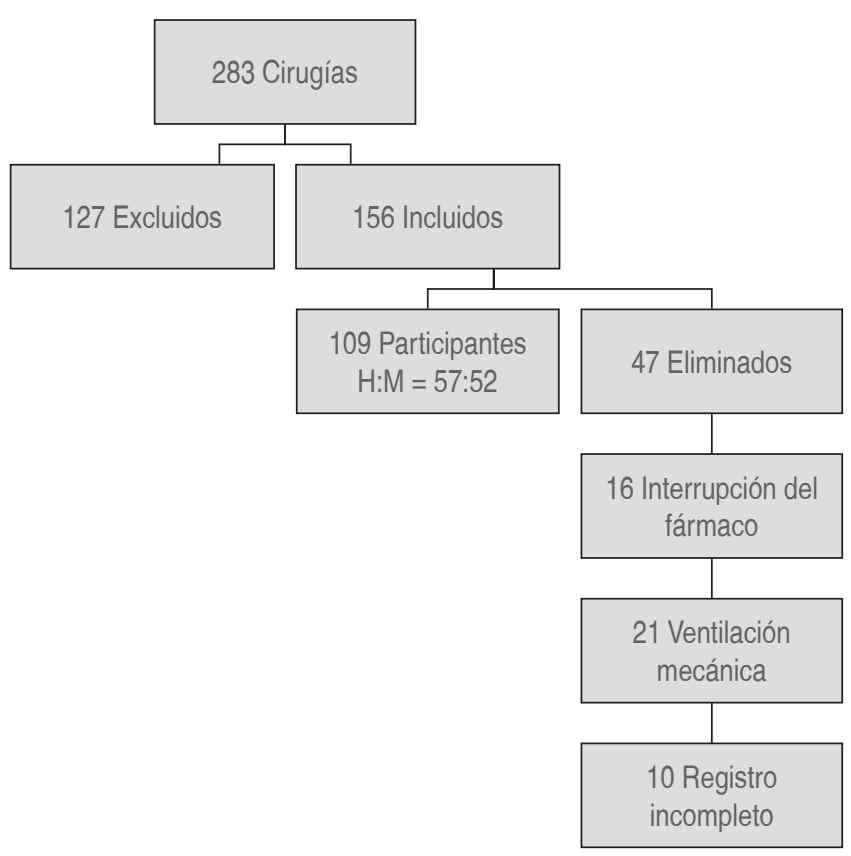

Figura 1: Grupo de estudio.

\begin{tabular}{lcccc} 
& Tabla 1: Datos demográficos y características clínicas. & \\
& & Error estándar & Mediana & Amplitud intercuantil 25-75\% \\
\hline Edad (meses) & $58.06 \pm 56.95$ & 5.45 & 25 & $11.5-101.5$ \\
Peso $(\mathrm{kg})$ & $21.4 \pm 18.7$ & 1.70 & $13-35$ & $8.3-28.5$ \\
Tiempo quirúrgico $(\mathrm{min})$ & $241.11 \pm 90.48$ & 8.66 & 240 & $180-280$ \\
Tiempo anestésico $(\mathrm{min})$ & $362.02 \pm 94.8$ & 9.08 & 360 & $300-400$ \\
Sangrado $(\mathrm{mL})$ & $434.67 \pm 427.15$ & 40.91 & 310 & $220-500$ \\
Tasa de fentanyl $(\mu \mathrm{g} / \mathrm{kg} / \mathrm{h})$ & $4.62 \pm 1.23$ & 0.11 & 4.4 & $3.8-5$ \\
Dosis total de fentanyl $(\mu \mathrm{g})$ & $527.77 \pm 523.4$ & 50.13 & 119 & $196.1-641$ \\
Total dosis de rescate & $1 \pm 1.29$ & 0.12 & $0-2$
\end{tabular}


Tabla 2: Análisis univariable de la población de estudio.

\begin{tabular}{|c|c|c|c|c|c|}
\hline & Control adecuado del dolor & Sin control del dolor & Total de la muestra & $\mathrm{p}$ & OR \\
\hline Pacientes n (\%) & $78(71.56)$ & $31(28.40)$ & & $<0.0001$ & \\
\hline Sexon $(\%)$ & & & & 0.17 & 1.8 \\
\hline Femenino & $41(78.84)$ & $11(21.15)$ & $52(47.7)$ & & \\
\hline Masculino & $37(64.10)$ & $20(35.00)$ & $57(52.2)$ & & \\
\hline ASA $n(\%)$ & & & & 0.97 & \\
\hline 1 & 3 & 1 & $4(3.6)$ & & \\
\hline 2 & 64 & 25 & $89(81.6)$ & & \\
\hline 3 & 11 & 5 & $16(14.6)$ & & \\
\hline Diagnóstico n (\%) & & & & 0.32 & \\
\hline Craneosinostosis & $38(65.50)$ & $20(34.40)$ & $58(53.2)$ & & \\
\hline Tumor de fosa posterior & $20(74.04)$ & $7(25.90)$ & $27(24.7)$ & & \\
\hline Craneofaringioma & $6(85.70)$ & $1(14.20)$ & $7(6.4)$ & & \\
\hline Otras & $14(82.35)$ & $3(17.60)$ & $17(15.5)$ & & \\
\hline Tipo de cirugía n (\%) & & & & 0.78 & 1.27 \\
\hline Supratentorial & $72(71.20)$ & $29(28.70)$ & $101(92.6)$ & & \\
\hline Infratentorial & $6(75.00)$ & $2(25.00)$ & $8(7.3)$ & & \\
\hline Tipo de anestesia & & & & 0.4 & 0.69 \\
\hline Anestesia total intravenosa & $51(75.0)$ & $17(25.00)$ & $68(62.3)$ & & \\
\hline Anestesia general & $27(65.80)$ & $14(34.15)$ & $41(37.6)$ & & \\
\hline Vómito $\mathrm{n}(\%)$ & & & & 0.33 & 2.6 \\
\hline Presencia & $1(3.13)$ & $6(7.79)$ & $7(6.4)$ & & \\
\hline Ausencia & $30(96.80)$ & $72(92.3)$ & $102(93.58)$ & & \\
\hline Náusea n (\%) & & & & 0.61 & 1.5 \\
\hline Presencia & $2(6.25)$ & $7(9.09)$ & $9(8.2)$ & & \\
\hline Ausencia & $29(93.54)$ & $71(91.0)$ & $100(91.7)$ & & \\
\hline
\end{tabular}

$\mathrm{n}=$ número de pacientes.

pacientes mayores de 11 años, se llevó a cabo un estudio de cohorte descriptivo, (observacional, prospectivo, longitudinal y sin grupos comparativos) en el que se incluyeron a todos los niños de 0-17 años programados para cirugía intracraneal electiva en un período de 24 meses de enero de 2015 a diciembre de 2017.

En los criterios de inclusión se consideró a todos los pacientes con estado físico de la Sociedad Americana de Anestesia (ASA por sus siglas en inglés) I-III y escala de coma de Glasgow de 15 puntos previo al evento quirúrgico. Dentro de los criterios de exclusión, se consideraron a niños que requirieron cirugía de urgencia, pacientes bajo tratamiento crónico con algún medicamento que pueda influir en el dolor postoperatorio (antiinflamatorios no esteroideos, opioides, antiepilépticos y neuromoduladores) y se eliminó a quienes se les interrumpió el tratamiento analgésico establecido o requirieron ventilación mecánica en el postoperatorio.

Durante el período intraoperatorio el anestesiólogo proporcionó anestesia total intravenosa (ATIV) con propofol y fentanyl en infusión o anestesia general balanceada, utilizando fentanyl en infusión y sevoflurano. En ambas técnicas anestésicas se administró fentanyl mediante bomba Medex (Medfusion 3500), realizando ATIV manual, calculando una concentración plasmática objetivo entre 0.004-0.006 $\mu \mathrm{g} / \mathrm{mL}$ durante el procedimiento quirúrgico. A todos los pacientes

\section{Control del dolor}

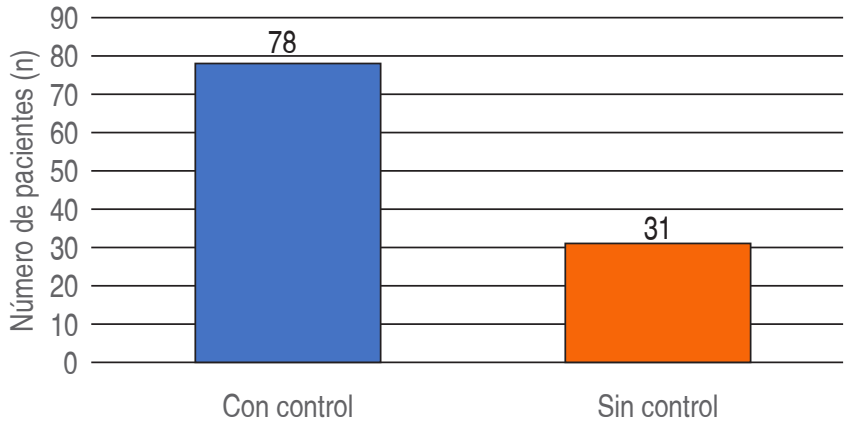

Figura 2: Comparación de pacientes en relación a control del dolor.

se les aplicó bloqueo de escalpe con bupivacaína al 0.25\% inmediatamente después de la inducción anestésica.

Para analgesia postoperatoria los pacientes recibieron buprenorfina $6 \mu \mathrm{g} / \mathrm{kg} / \mathrm{día}$ + ketorolaco $2 \mathrm{mg} / \mathrm{kg} /$ día + ondansetrón $0.3 \mathrm{mg} / \mathrm{kg} / \mathrm{día}$ en infusión continua intravenosa mediante bomba elastomérica para 30 horas, la cual se inició al ingresar a la unidad de terapia intensiva pediátrica.

Los investigadores realizaron evaluaciones al inicio de la infusión a las cuatro, ocho, 12, 24 y 30 horas, que abarcaron 
parámetros hemodinámicos tales como frecuencia cardíaca, tensión arterial y saturación de oxígeno así como valoración del dolor mediante diferentes escalas de acuerdo a la edad del paciente. En los niños menores de cuatro años se aplicó la escala Face-Legs-Activity-Cry-Consolability (FLACC) ${ }^{(9)}$, en los niños de cinco a siete años la Escala Facial de Dolor de Wong-Baker ${ }^{(10,11)}$ y en mayores de siete años la escala visual analógica (EVA) ${ }^{(11)}$. Las tres escalas se evalúan estableciendo un valor numérico entre 0 -10, tomando como punto de corte un valor $\geq 4$ puntos. Asimismo, se registró la presencia de eventos adversos y el puntaje de la escala de Ramsay para evaluar presencia y profundidad de sedación.

En el análisis estadístico se describió la distribución univariada con todas las variables; en caso de que la discrepancia ante el modelo normal se considere importante, se reportó la mediana y amplitud intercuartil. Para las variables cuantitativas se describió su resumen por media y desviación estándar. Con respecto a las variables cualitativas se reportaron las frecuencias absoluta y relativa. Para las escalas de dolor y la escala de Ramsay se establecieron los casos con dolor intenso y sedación profunda de acuerdo con los puntos de cortes establecidos para cada escala. Se determinó la correlación entre las variables de dolor con las variables hemodinámicas por medio del coeficiente de correlación de Spearman. Se evaluó la asociación entre las variables preoperatorias (edad, sexo y tipo de cirugía) con las escalas de dolor por el modelo lineal general. Los parámetros estimados se reportaron con el intervalo de confianza de $95 \%$ y la significancia estadística se reconocerá al nivel de $\alpha<0.05$. Con las asociaciones analizadas estadísticamente no significativas se realizó el análisis de poder post-hoc, estableciendo como criterio del nivel de poder estadístico suficientemente alto1- $\beta>0.8$. Todos los análisis estadísticos se hicieron con el paquete estadístico comercial JMP10 de SAS Institute, Inc.

\section{RESULTADOS}

Durante el período establecido, 283 pacientes fueron sometidos a craneotomías, de los cuales 127 pacientes fueron excluidos. Se incluyeron en la muestra del estudio 156 pacientes, de los que se eliminaron 47 (Figura 1).

\begin{tabular}{lcccc}
\multicolumn{5}{c}{ Tabla 3: Relación de efectos secundarios. } \\
Variable & Náusea & $p<0.05$ & Vómito & $p<0.050$ \\
\hline Sexo & Hombres & 0.837 & Hombres & 0.790 \\
Diagnóstico & Otros & 0.126 & Otros & 0.028 \\
Tipo de rescate & Combinado & 0.177 & Combinado & 0.376 \\
ASA & III & 0.254 & III & 0.1424 \\
Anestesia & TIVA & 0.302 & TIVA & 0.158 \\
Dolor & No controlado & 0.614 & Controlado & 0.334
\end{tabular}

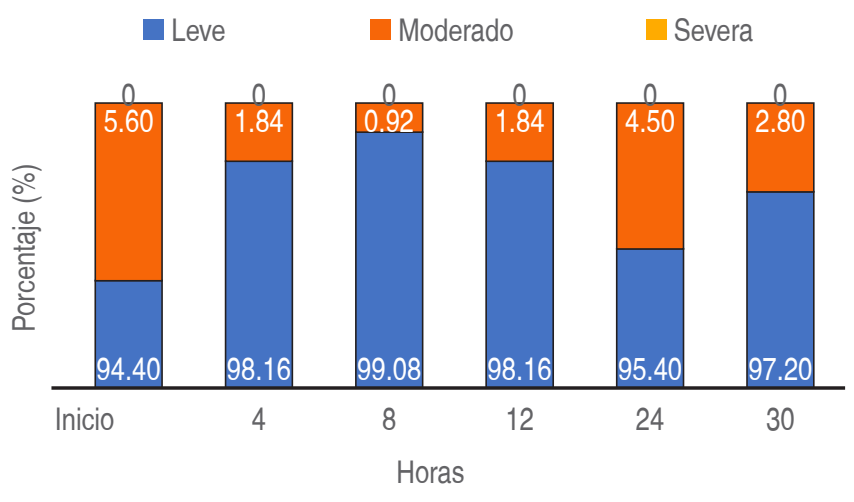

Figura 3. Grado de sedación mediante la escala de Ramsay, durante la infusión de buprenorfina y ketorolaco para manejo del dolor postoperatorio.

De los 109 pacientes estudiados, 52 fueron femeninos y 57 masculinos. La edad media fue de cuatro años ocho meses, con un rango de tres meses a 16 años. El peso promedio fue de $21.4 \mathrm{~kg}$ (Tabla 1).

Los diagnósticos de los pacientes incluyeron: craneosinostosis 58 (53.2\%), tumores de fosa posterior 27 (24.7\%), craneofaringioma 7 (6.4\%), otros supratentoriales (tumores interventriculares, lesiones en cíngulo e hidrocefalias) 17 (15.5\%). El tipo de cirugía que más se realizó fue la supratentorial (Tabla 2).

Al tomar en consideración el puntaje en las escalas, junto con el comportamiento hemodinámico a las cuatro, ocho, 12, 24 y 30 horas de la infusión se observó que con la combinación de buprenorfina-ketorolaco hubo un adecuado control del dolor en 78 pacientes (71.56\%), mientras que en 31 (28.4\%) se encontró inadecuado control del dolor, con una diferencia estadísticamente significativa $(\mathrm{p}<0.001)$ (Figura 2 y Tabla 2).

La evaluación del grado de sedación se realizó utilizando la escala de Ramsay. Se observó sedación moderada en 5.6\% de los pacientes al inicio de la infusión y a las 24 horas (4.5\%), sin repercusión significativa en las variables hemodinámicas como FC, saturación y tensión arterial. Sólo se reportó incidencia de un paciente con somnolencia (0.9\%) sin requerir administración de fármaco antagonizante de opioide o maniobras adicionales (Figura 3 ).

Dentro de los efectos colaterales se encontró náusea en 9 pacientes (8.2\%) y vómito en 7 (6.64\%); no se presentó prurito, retención urinaria, sedación profunda, depresión respiratoria ni alteración neurológica (Figura 4 y Tabla 3).

Para el análisis univariable se dividió a la población en dos grupos: grupo 1: pacientes con control adecuado del dolor, grupo 2: pacientes sin control del dolor. El análisis univariable mostró una $\mathrm{p}>0.05$ en las variables de género, estado físico de ASA, diagnóstico, tipo de cirugía, náusea y vómito, no encontrando diferencia significativa en éstas (Tabla 2). 
En ambos sexos hubo adecuado control del dolor, con un porcentaje mayor en el sexo femenino. Con respecto al estado físico de ASA, se notó una relación directamente proporcional entre control inadecuado del dolor y mayor puntaje en el estado físico del ASA.

En relación al tipo de cirugía se apreció menor control del dolor en los pacientes sometidos a remodelación craneal por craneosinostosis y cirugía supratentorial (Tabla 2).

Con respecto a la técnica anestésica, se observó un mejor control del dolor, en los que recibieron anestesia total intravenosa.

El tiempo total de la infusión fue de 30 horas, observándose que a las cuatro y 24 horas, los pacientes mostraron mayor puntaje en las escalas de dolor, sin relación con descontrol de las variables hemodinámicas, es decir, no se detectó hipertensión, desaturación ni taquicardia (Figura 5). El puntaje superior a las cuatro horas puede ser debido a presencia de irritabilidad o ansiedad, ya que no se observó descontrol en las variables hemodinámicas.

A los pacientes registrados con inadecuado control del dolor (31 pacientes), se les dio dosis de rescate con buprenorfina IV y/o paracetamol, previamente valorado por el médico intensivista tratante.

Concerniente a los rescates otorgados a los pacientes con un control inadecuado, se notó que fueron necesarios a partir de las 20 horas de la infusión (Figura 6). El número de rescates fueron de 1-3, con una media de 2. La población que recibió rescates con buprenorfina IV fueron 14 (12.8\%), 5 (4.5\%) con paracetamol IV y 9 (8.2\%) con ambos fármacos. Tres pacientes $(2.7 \%)$ obtuvieron una calificación en las escalas de dolor de 6, en una sola ocasión, por lo que se catalogaron como «sin control del dolor»; sin embargo, no recibieron rescates adicionales y en el resto de las evaluaciones obtuvieron una calificación de 0 en las escalas de dolor.

A pesar del uso de rescates con buprenorfina no se encontró sedación profunda en los pacientes.

Los niños que requirieron mayor número de rescates fueron aquéllos con diagnóstico de craneosinostosis y cirugía supratentorial.

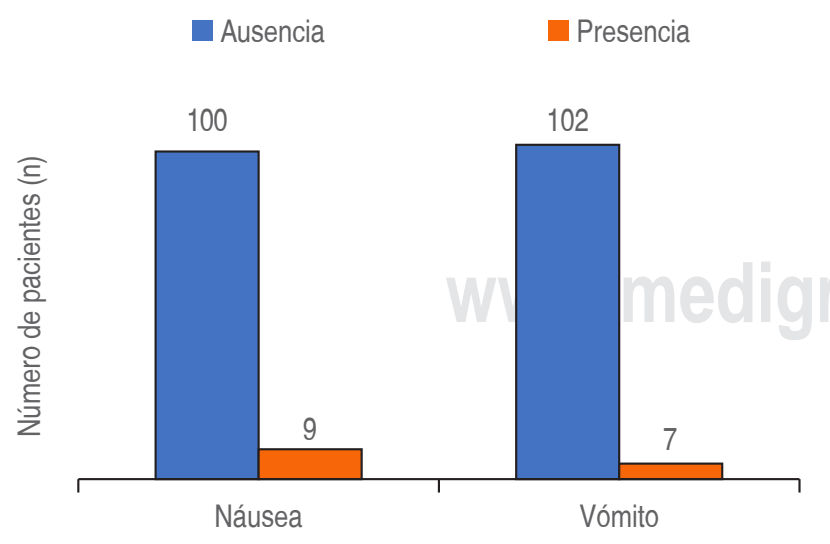

Figura 4: Frecuencia de principales efectos secundarios.

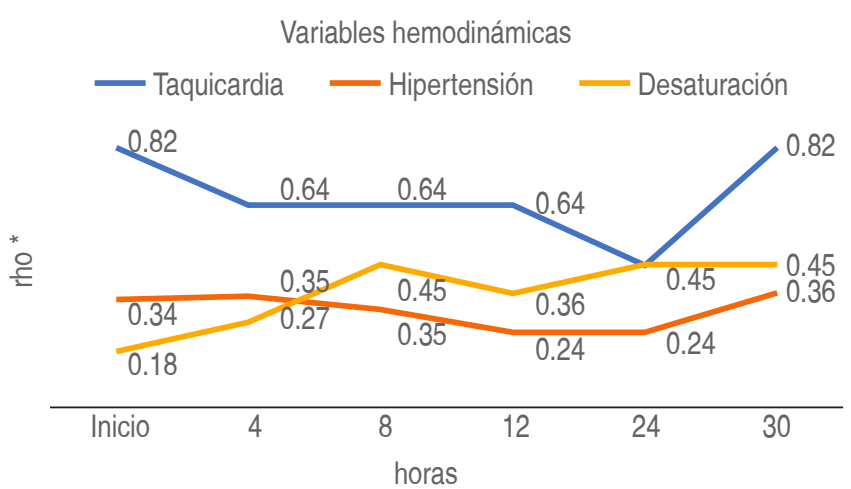

Figura 5: Comportamiento de variables hemodinámicas durante la infusión. *Coeficiente de correlación: +1 = correlación positiva, 0 = sin correlación, $-1=$ correlación negativa.

\section{DISCUSIÓN}

Existe controversia en cuanto al uso de opioides para manejo del dolor postoperatorio de pacientes neuroquirúrgicos, por una parte, algunos anestesiólogos se oponen al uso de opioides debido al riesgo de efectos adversos, pero por otra parte, se ha reportado la presencia de hipertensión arterial, agitación, vómitos, temblores y ansiedad en aquellos pacientes con control inadecuado del dolor, lo que puede condicionar aumento de la presión intracraneal y hemorragia, mermando la recuperación y el resultado postquirúrgico.

Hay evidencia en varios estudios prospectivos en adultos que confirman la experiencia de dolor moderado a severo en aproximadamente $60 \%$ de los pacientes después de una craneotomía, que puede persistir más allá del período postoperatorio inmediato ${ }^{(3,7,12-14)}$.

En la actualidad encontramos pocos estudios enfocados en evaluar la incidencia y severidad del dolor postoperatorio en pacientes pediátricos después de cirugía craneal ${ }^{(4,15)}$, así como un número limitado de estudios que reporten el uso de opioides en niños sometidos a craneotomías.

Los opioides parenterales constituyen la piedra angular para el manejo del dolor moderado a severo, especialmente después de cirugía mayor. El dolor nociceptivo suele responder bien a los opioides.

Algunos estudios publicados en población pediátrica han examinado el uso de opioides para craneotomías, entre ellos: fentanyl-midazolam ${ }^{(8)}$, tramadol-fentanyl ${ }^{(16)}$, morfina ${ }^{(17)}$, codeína $^{(18)}$, hidromorfona, oxicodona ${ }^{(19)}$, fentanyl, tramadol, morfina ${ }^{(20)}$ con resultados variables, pero ninguno que documente el uso de buprenorfina, ketorolaco y ondansetrón para este tipo de intervención quirúrgica.

Nuestro estudio de cohorte observacional prospectivo reveló una alta incidencia de control adecuado de dolor postquirúrgico (78\%) con la combinación de buprenorfina y ketorolaco en infusión. La administración en infusión continua 
permite a los pacientes tratar su dolor sin la presencia de picos y valles farmacológicos, ya que mantiene una concentración plasmática continua. La administración sistémica intermitente de y PRN (Pro re nata) ha sido un método estándar para la analgesia postoperatoria ${ }^{(1,3)}$, pero estas estrategias pueden ocasionar períodos de sobresedación (efecto opiáceo pico), seguido de períodos de analgesia inadecuada (efecto opiáceo final) $)^{(1)}$.

Maxwell y colaboradores realizaron un estudio donde se evaluaron las terapias analgésicas de tres hospitales pediátricos universitarios de Estados Unidos, reportando en uno de los centros el uso de hidromorfona, fentanyl o morfina en infusión IV, concomitante con una infusión de naloxona a dosis baja (1-1.5 $\mu \mathrm{g} / \mathrm{kg} / \mathrm{h}$ ) como profilaxis para limitar los efectos colaterales inducidos por los opioides ${ }^{(19)}$. En nuestro estudio no se apreció sedación profunda con el uso de buprenorfina que interfiriera en el examen neurológico, sólo se notó un paciente con somnolencia que no requirió administración de fármaco antagonizante de opioides. Se observó la presencia de sedación moderada al inicio de la infusión y a las 24 horas. La concentración plasmática promedio de fentanyl al final de la cirugía fue de $0.00462 \mu \mathrm{g} / \mathrm{mL}$, dosis adecuada para el inicio de la infusión, permitiendo una continuidad del manejo del dolor a base de opioide. La presencia de sedación moderada al inicio de la infusión puede ser por el sinergismo con el efecto residual de fentanyl. En estudios posteriores se debe evaluar la causa desencadenante del pico de dolor a las 24 horas. Aunque más que dolor, se puede tratar de cierto grado de irritabilidad, ya que no hubo cambios en las constantes vitales. Tampoco requirieron administración de naloxona los pacientes que recibieron rescates de buprenorfina IV.

Otro de los efectos adversos temidos de los opioides es náusea y vómito. La presencia de náusea y vómito en nuestro estudio es muy baja, 8.2 y 6.64\% respectivamente, a diferencia

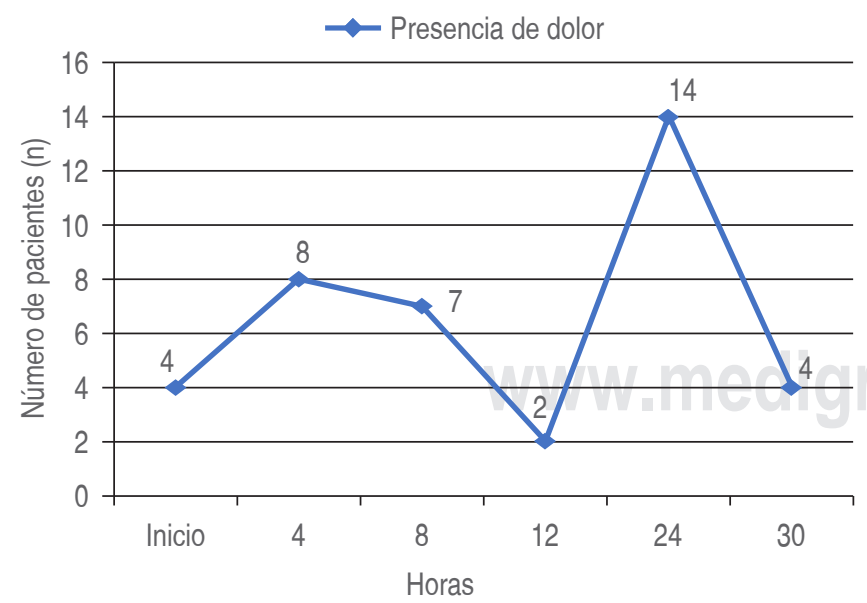

Figura 6: Comportamiento del dolor durante la infusión analgésica en pacientes con control inadecuado. de la reportada en otros centros hospitalarios con el uso de opioides para analgesia: The Johns Hopkins Hospital (náusea 56\% y vómito 33\%); The Boston Children's Hospital (náusea 53\% y vómito 58\%); The Children's Hospital of Philadelphia (náusea $42 \%$, vómito $66 \%)^{(19)}$. Esto nos indica que al administrar ondansetrón en infusión continua para mantener una dosis constante podemos reducir la incidencia de náusea y vómito al utilizar opioides en este tipo de cirugías. En el estudio realizado por Xing y colaboradores ${ }^{(3)}$ se compararon tres regímenes de analgesia postoperatoria para craneotomías que incluyó fentanyl, morfina y tramadol así como un grupo control al que se le administró solución salina. Todas las bombas analgésicas se prepararon para 50 horas y contenían además $0.4 \mathrm{mg} / \mathrm{kg}$ de ondansetrón. En este estudio se reporta una incidencia de náusea de $27.5 \%$ y vómito de $47.5 \%$ en el grupo que recibió tramadol. Nosotros encontramos una incidencia muy baja de náusea y vómito postoperatorio al utilizar buprenorfina en combinación con ondansetrón, lo que puede sugerir que, además de ser importante la administración en infusión continua, también influye la dosis del antiemético así como el opioide que se elija en este tipo de cirugías.

Una de las estrategias actuales para abordar el control del dolor es la analgesia multimodal. Este concepto implica que la asociación de diferentes intervenciones analgésicas por diferentes vías, consiguen una efectividad mayor sobre su utilización única, disminuye los efectos secundarios y aumenta la satisfacción de los pacientes, especialmente porque los efectos de un enfoque multimodal se consideran sinérgicos más que aditivos ${ }^{(21)}$. La analgesia multimodal se recomienda para el manejo del dolor perioperatorio pediátrico ${ }^{(22)}$. En este estudio se añadió la infusión ketorolaco, lo que permite disminuir dosis y en consecuencia efectos secundarios, otra estrategia multimodal incluyó el uso de bloqueo de escalpe previo a la insición quirúrgica.

Uno de los posibles riesgos del uso de antiinflamatorios no esteroideos (AINEs) en neurocirugía craneal es la disminución de la función plaquetaria, lo que podría condicionar hemorragia. No obstante, ninguno de los pacientes en nuestro estudio presentó sangrado postoperatorio. En estudios previos se ha valorado el uso de ketorolaco en infusión y por horario establecido durante las primeras 24 horas del postoperatorio sin detectar hemorragia postoperatoria como evento adverso ${ }^{(5,23)}$. Sin embargo, para establecer un perfil de seguridad de los AINEs en este tipo de cirugías es necesario un mayor número de pacientes.

Referente al tipo de cirugía, encontramos menor control del dolor en las cirugías supratentoriales, lo cual se reflejó en las escalas de dolor y en el requerimiento de dosis de rescate con buprenorfina; sin embargo, en la población de estudio hubo mayor número de pacientes con este tipo de abordaje.

La buprenorfina es de interés en el control del dolor postoperatorio y oncológico pediátrico debido a su larga duración de acción y metabolismo en gran parte independiente de la función renal, aunque son escasamente descritos los parámetros 
farmacocinéticos y farmacodinámicos en niños ${ }^{(24)}$. Una revisión retrospectiva de sobredosis de buprenorfina en 54 niños reportada por los centros de envenenamiento de Estados Unidos revela que los niños que ingirieron $>2 \mathrm{mg}$ de buprenorfina tuvieron más probabilidades de experimentar algún efecto clínico, ningún niño que ingirió $<4 \mathrm{mg}$ experimentó un efecto severo $^{(25)}$. Gracias al amplio rango de seguridad de buprenorfina de presentar eventos adversos es una opción adecuada en craneotomías, ya que se desea evitar sobresedación, náusea y vómito que interfiera con una valoración neurológica temprana.

$\mathrm{Al}$ administrar los fármacos en una infusión disminuimos costos, puesto que otorgarlos por separado implica la disponibilidad de tres bombas para cada paciente, lo cual en el contexto de varios hospitales de Latinoamérica es complicado.

Este esquema analgésico se ha utilizado por varios años en nuestro centro de trabajo, por lo que consideramos pertinente realizar este estudio para observar los alcances y limitaciones de dicha terapia analgésica.

\section{CONCLUSIONES}

El dolor postcraneotomía en pediatría es un problema clínico desafiante, a través de una estrategia multimodal se puede obtener un alivio apropiado del dolor. Los resultados del actual estudio sugieren que el uso de buprenorfina, ketorolaco y ondansetrón en infusión es una opción efectiva para tratar el dolor en este tipo de pacientes, debido a que no ocasionó depresión respiratoria o sedación excesiva que imposibilite una valoración neurológica adecuada, además la presencia de náusea y vomito fue muy baja.

Se requiere un tamaño mayor de muestra para obtener mejor validez del estudio y compararlo con otras terapias analgésicas.

\section{REFERENCIAS}

1. Ortiz-Cardona J, Bendo A. Perioperative pain management in the neurosurgical patient. Anestesiology Clin. 2007;25:655-674.

2. Headache Classification Committee of the International Headache Society. The International Classification of Headache Disorders. Vol. 33, 3a ed. Cephalalgia 2013, 629-808.

3. Tsaousi GG, Logan SW, Bilotta F. Postoperative pain control following craniotomy: a systematic review of recent clinical literature. Pain Pract. 2017;17:968-981.

4. Bronco A, Pietrini D, Lamperti M, Somaini M, Tosi F, Miguell del Lungo L, et al. Incidence of pain after craniotomy in children. Pediatric Anesthesia. 2014;24:781-787.

5. Na HS, An SB, Park HP, Lim YJ, Hwang JW, Jeon YT, et al. Intravenous patient-controlled analgesia to manage the postoperative pain in patients undergoing craniotomy. Korean J Anesthesiol. 2011;60:30-35.

6. Verchere E, Grenier B, Mesli A, Siao D, Sesay M, Maurette P. Postoperative pain management after supratentorial craniotomy. J Neurosurgical Anesthesiology. 2002;14:96-101.

7. Hansen M, Brennum J, Moltke F, Dahl J. Pain treatment after craniotomy: where is the (procedure-specific) evidence? A qualitative systematic review. Eur J Anaesthesiol. 2011;28:821-829.

8. Chiaretti A, Genovese O, Antonelli A, Tortorolo L, Ruggiero A, Focarelli $\mathrm{B}$, et al. Patient-controlled analgesia whit fentanil and midazolam in children with postoperative neurosurgical pain. Childs Nerv Syst. 2008;24:119-124.

9. Malviya S, Voepel-Lewis T, Burke C, Merkel S, Tait AR. The revised FLACC observational pain tool: improve reliability and validity for pain assessment in children with cognitive impairment. Paediatr Anaesth. 2006;16:258-265.

10. Stinson JN, Kavanagh T, Yamada J, Gill N, Stevens S. Systematic review of the psychometric properties, interpretability and feasibility of selfreport pain intensity measures for use in clinical trials in children and adolescents. Pain. 2006;125:143-157.

11. Malmierca F, Pellegrini J, Malmierca A. Valoración del dolor en pediatría. En: Dolor en pediatría, Madrid: Ergon; 2008, pp. 1-17.

12. Mordhorst C, Latz B, Kerz T, wiser G, Schmidt A, Schneider A, Jahn-Eimermacher A, Wener C, Engelhard K. Prospective assessment of postoperative pain after craniotomy. J Neurosurg Anesthesiol. 2010;22:202-206.
13. Morad A, Winters B, Stevens R, White E, Weingart J, Yaster M, Gottschalk A. The efficacy of intravenous patient-controlled analgesia after intracranial surgery of the posterior fossa: A prospective, randomized controlled trial. Anesth Analg. 2012;114:416-423.

14. Gottschalk A, Berkow L, Stevens R, Mirski M, Thompson R, White E, et al. Prospective evaluation of pain and analgesic use following major elective intracranial surgery. J Neurosurg. 2007;106:210-216.

15. Teo JH, Palmer GM, Davidson AJ. Post-craniotomy pain in a paediatric population. Anaesth Intensive Care. 2011;39:89-94.

16. Chiaretti A, Viola L, Pietrini D, Piastra M, Savioli A, Tortorolo L, et al. Preemptive analgesia with tramadol and fentanil in pediatric neurosurgery. Childs Nerv Syst. 2000;16:93-99.

17. Warren D, Bowen-Roberts T, Ou C, Purdy R, Steinbok P. Safety and efficacy of continuous morphine infusions following pediatric cranial surgery in a surgical ward setting. Childs Nerv Syst. 2010;26:1535-1541.

18. Roberts GC. Post-craniotomy analgesia: current practices in British Neurosurgical Centres -a survery of post-craniotomy analgesic practices. Eur J Anaesthesiol. 2005;22:328-332.

19. Maxwell LG, Buckley GM, Kudchadkar SR, Ely E, Stebbins EL, Dube C, et al. Pain management following major intracranial surgery in pediatric patients: a prospective cohort study in three Academic Children's Hospitals. Paediatr Anaesth. 2014;24:1132-1140.

20. Xing F, Xin L, Shan F, Mei C, Fan Y. Postoperative analgesia for pediatric craniotomy patients: a randomized controlled trial. BMC Anesthesiology. 2019;19:2-10.

21. Ban V, Bhoja R, McDonagh D. Multimodal Analgesia for craniotomy. Curr Opin Anesthesiol. 2019;32:592-599.

22. Howard R, Carter B, Curry J, Morton N, Rivett K, Rose M, et al. Good practice in postoperative and procedural pain management. A guideline from the Association of Paediatric Anaesthetists of Great Britain and Ireland. Pediatric Anesth. 2012;22:1-79.

23. Ready LB, Brown CR, Stahlgren LH, Egan KJ, Ross B, Wild L, et al. Evaluation of intravenous ketorolac administered by bolus or infusion for treatment of postoperative pain. Anesthesiology. 1994;80:1277-1286.

24. Michel E, Anderson B, Zernikow B. Buprenorphine TTS for children- a review of the drug's clinical pharmacology. Pediatric Anesthesia. 2011;21:280-290.

25. Hayes B, Klein-Schwartz W, Doyon S. Toxicity of buprenorphine overdoses in children. Pediatrics. 2008;121:e782-786. 